Check for updates

Cite this: Mater. Adv., 2021, 2, 2906

Received 4th March 2021 Accepted 5th April 2021

DOI: 10.1039/d1ma00189b

rsc.li/materials-advances

\section{MXenes and MXene-based materials for tissue engineering and regenerative medicine: recent advances}

\begin{abstract}
Siavash Iravani (D) *a and Rajender S. Varma (D) ${ }^{b}$
In view of their unique planar structure and outstanding physical and chemical properties, twodimensional (2D) materials have garnered the attention of interdisciplinary researchers in the domain of biomedical and clinical applications. MXenes are 2D transition metal carbides and nitrides with outstanding characteristics, comprising huge surface area, biocompatibility, low toxicity, significant electrical conductivity, antibacterial activity and hydrophilicity. Although numerous investigations have demonstrated the promising potential of MXenes for different biomedical applications that include biosensing, bioimaging, cancer therapy, tissue engineering, regenerative medicine, and drug delivery, there are still important challenging issues pertaining to their stability in physiological environments, sustained/controlled release of drugs, and biodegradability. The well-designed ultra-thin MXene nanosheets are deployable as promising biocompatible inorganic nanoplatforms for assorted biomedical applications via the clinical translation of nanomedicine; MXenes are good candidates for tissue engineering and regenerative medicine. Herein, recent progresses on 2D MXenes for state-of-the-art tissue engineering and regenerative medicine are discussed with emphasis on the significant challenges and future perspectives.
\end{abstract}

\section{Introduction}

A wide variety of materials, comprising ceramics, synthetic polymers and natural biopolymers have been commissioned for tissue engineering and regenerative medicine purposes. In this regard, nature-based nanomaterials and biopolymers such as animal- or plant-derived proteins or carbohydrates have displayed prominent roles in affecting cell activities, especially in terms of biocompatibility and chemical signals; ${ }^{1-3}$ their efficiency being restricted by quick biodegradability and inadequate mechanical features. However, post-spinning crosslinking procedures with appropriate cross linkers can be deployed to overcome these drawbacks; blending polymers with biocompatible synthetic polymers is a useful strategy for developing ideal scaffolding materials for tissue engineering applications. $^{3-6}$

MXenes are two-dimensional (2D) transition metal carbides and nitrides, where $\mathrm{Ti}_{3} \mathrm{C}_{2} \mathrm{~T}_{x}$ exemplifies the first candidate prepared by etching an $\mathrm{Al}$ layer in $\mathrm{Ti}_{3} \mathrm{AlC}_{2}, \mathrm{~T}_{x}$ being the surface

\footnotetext{
${ }^{a}$ Faculty of Pharmacy and Pharmaceutical Sciences, Isfahan University of Medical Sciences, Isfahan, Iran. E-mail: siavashira@gmail.com

${ }^{b}$ Regional Centre of Advanced Technologies and Materials, Department of Physical Chemistry, Faculty of Science, Palacký University in Olomouc, Šlechtitelů 27,

78371 Olomouc, Czech Republic
}

groups (e.g., $-\mathrm{F}$ and $-\mathrm{OH})$ generated during the etching process (Table 1). ${ }^{7,8}$ MXenes and MXene-based materials with remarkable electronic conductivity, elastic mechanical strength, hydrophilicity, and chemical stability have promising potentials for deployment in the field of environmental science/water treatment, energy storage, composite materials, drug/gene delivery, and biomedical/pharmaceutical sciences, ${ }^{9-14}$ and likewise have been utilized for biosensing, antibacterial materials, bioimaging, theranostic nanomedicine, and cancer diagnosis/ therapy. ${ }^{8,15-19}$ They have been employed in biosensing and bioimaging comprising magnetic resonance and photoacoustic imaging techniques in view of their unique tunable optical and magnetic properties. Similar interesting potentials have been uncovered as efficient agents for environmental and antimicrobial treatments due to their pollutant adsorption and appropriate antibacterial properties. ${ }^{20-22}$

The conventional organic compounds with acceptable biocompatibility and biodegradability attributes were widely investigated for biomedical applications, but their poor thermal and chemical stability and solitary functionality are the main shortcomings restricting their development in clinical terms. ${ }^{33}$ On the other hand, 2D structures fabricated via bottom-up (e.g., template method, hydro/solvothermal synthesis, microwave-assisted synthesis, chemical vapor deposition, and topochemical transformation approach) and 
top-down (e.g., ion-change, ultrasonic, and micromechanical exfoliation) tactics have shown attractive properties for biomedical applications. $^{34,35}$ As an example, selenium-coated tellurium nanoheterojunctions with nontoxicity advantages exhibited promising results for the eradication of hepatocellular carcinoma and lung cancer in preclinical models. In this study, the deployed photothermal therapy (PTT)-based approach could alter the microenvironment of tumors and stimulate significant apoptosis of cancerous cells. ${ }^{36}$ Additionally, black phosphorus nanosheets with large surface area, intrinsic photoacoustic features, biocompatibility, and biodegradability are potential candidates for diverse biomedical applications, including photothermal/photodynamic therapy, diagnostics imaging, biosensing, cancer diagnosis/therapy, drug/gene delivery, neuronal regeneration, and three dimensional (3D) printing scaffolds. ${ }^{37-39}$ For instance, to synergistically improve the efficacy of cancer therapy and management, a photothermal therapy tactic using black phosphorus was employed together with an anti-CD47 antibody-based immunotherapy strategy. ${ }^{40}$ It was indicated that the ablation of tumors via black phosphorus-based photothermal therapy stimulated the immunological responses specifically (local and systemic immune responses against cancers) and improved the inherently poor immunogenicity of tumors, enhancing the efficacy of cancer immunotherapy, providing synergistic influences to suppress the progression of tumors, and stimulating abscopal consequences to inhibit metastatic cancer. ${ }^{40}$ Despite the attractive

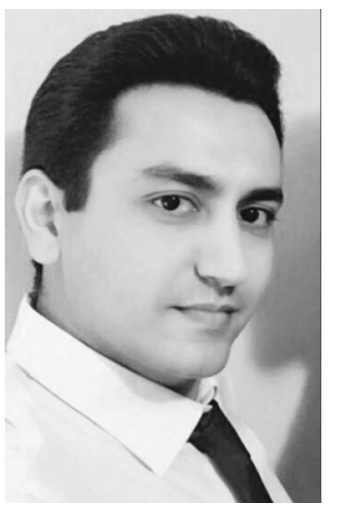

Siavash Iravani
Dr Iravani has worked on several academic research projects at the Isfahan University of Medical Sciences (Faculty of Pharmacy and Pharmaceutical Sciences), including green and eco-friendly synthesis of nanomaterials, plant-derived nanostructures, phytochemical analysis, graphene-based nanocomposites, water treatment technologies, nanoparticles for drug delivery in cancer, nanocarriers, and drug nanoparticles. His previous experience, of more than ten years, centers on drug development and industrial pharmacy in various capacities including research and development, formulation, and quality control. Dr Iravani has authored over 70 peer-reviewed scientific publications including 15 book chapters and two scientific books. advantages of black phosphorus, the intrinsic instability may restrict its applications, causing rapid ambient degradation. ${ }^{37,38,41}$ This can be considered as one of the important challenges for commercial/up-scalable production. ${ }^{42}$ Compared with these 2D materials, MXenes and MXene-base materials with high hydrophilicity, biodegradability, chemical stability, thermal/electrical conductivity, sorption selectivity, biocompatibility, and antibacterial activity are good candidates in the field of tissue engineering and regenerative medicine. ${ }^{43}$ Herein, significant recent advancements pertaining to the tissue engineering and regenerative medicine applications of MXenes have highlighted as well as their important challenges and future perspectives.

\section{Synthesis of MXenes}

MXenes have been primarily used for the improvement of mechanical characteristics and electrical conductivities of polymer matrixes. ${ }^{44-46}$ These materials can be utilized as $2 \mathrm{D}$ inorganic filling materials to modify the surface attributes of electrospun nanofibers for biomedical purposes. During the preparative process of MXenes, the hydrofluoric acid etching procedure increased the hydrophilic functional groups (e.g., $\mathrm{OH}, \mathrm{O}$, and $\mathrm{F}$ ) on their exteriors that determine their enhanced hydrophilic characteristics, leading to improved biocompatibility. ${ }^{47}$ Additionally, the fabrication of MXenes from relatively inert metals

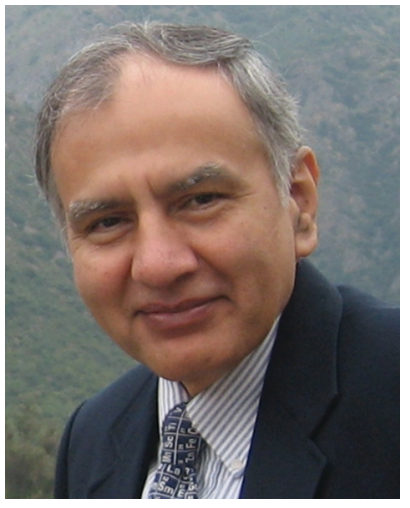

Rajender S. Varma
Prof. Varma (H-Index 113, Highly Cited Researchers 2016, 18, 19, 20; Publons Awardee 2018, 2019) was born in India (PhD, Delhi University 1976). After postdoctoral research at Robert Robinson Laboratories, Liverpool, U.K., he was a faculty member at Baylor College of Medicine and Sam Houston State University prior to joining the Sustainable Technology Division at the U.S. Environmental Protection Agency in 1999. He has a visiting scientist's position at Regional Centre of Advanced Technologies and Materials, Palacky University at Olomouc, Czech Republic. He has over 45 years of research experience in management of multidisciplinary technical programs ranging from natural products chemistry to development of more environmentally friendly synthetic methods using microwaves, ultrasound, etc. Lately, he is focused on greener approaches to assembly of nanomaterials and sustainable applications of magnetically retrievable nanocatalysts in benign media. He is a member of the editorial advisory board of several international journals, has published over 670 papers, and has been awarded 17 U.S. Patents, 6 books, 26 book chapters, and 3 encyclopedia contributions with 46500 citations. 
Table 1 Some important mechanical, electronic, magnetic, and optical properties of MXenes

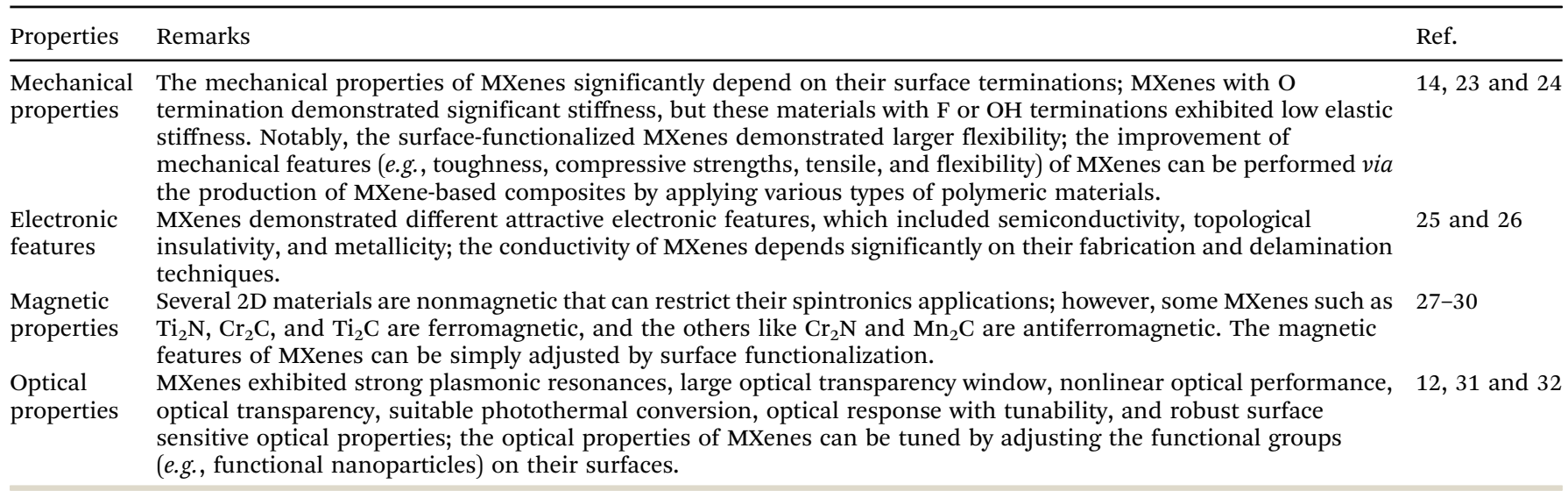

in combination with carbonitrides would lend support to their cell-friendly conduct during cell proliferation, adhesion, and differentiation processes. ${ }^{18,47}$ For example, electrospun $\mathrm{Ti}_{3} \mathrm{C}_{2} \mathrm{~T}_{z}$ (MXene)/chitosan composite fibers have been fabricated for antibacterial activity against Escherichia coli and Staphylococcus aureus, encompassing wound healing functions. ${ }^{48}$ In a cancer theranostics study, superparamagnetic $2 \mathrm{D} \mathrm{Ti}_{3} \mathrm{C}_{2}$ MXenes were assembled exploiting the surface chemistry of specific MXenes via in situ growth of superparamagnetic $\mathrm{Fe}_{3} \mathrm{O}_{4}$ nanomaterials on the MXene's surface. ${ }^{49}$ The ensuing magnetic composite form of MXene showed an elevated $T_{2}$ relaxivity of $394.2 \mathrm{mM}^{-1} \mathrm{~s}^{-1}$ and proficient contrast-improved magnetic resonance imaging (MRI) of tumors, offering apt potentials for therapeutic direction. These MXenes with superparamagnetic attributes demonstrated remarkable photothermal transformation competence (48.6\%) for the successful photothermal killing of cancerous cells and ablation of tumor tissues, in vitro and in vivo. Notably, these MXene-based composites with acceptable biocompatibility can be applied in the field of theranostic nanomedicine, and further study should be undertaken for their fabrication and evaluation. ${ }^{49}$

MXenes can be fabricated by the exfoliation of an "A" layer from a "MAX" antecedent; where $\mathrm{M}, \mathrm{A}$, and $\mathrm{X}$ represent the transition metal, elements from IIIA or IVA, and $\mathrm{N}$ or $\mathrm{C}$, respectively. ${ }^{14,46,50,51}$ These $2 \mathrm{D}$ materials have been produced via different strategies such as the urea glass method, ${ }^{52}$ chemical vapor deposition, ${ }^{53}$ molten salt etching, ${ }^{54}$ hydrothermal syntheses, ${ }^{55}$ and electrochemical fabrication. ${ }^{56}$ Bottom-up chemical vapor deposition and top-down wet etching tactics have been broadly developed for the synthesis of MXenes. ${ }^{57}$ The wet etching technique can be applied to provide MXenes with high quality and the presence of terminations, causing the fabrication of MXenes with a basically hydrophilic nature. ${ }^{58}$ As an example, $\mathrm{Ti}_{3} \mathrm{C}_{2} \mathrm{~T}_{x}$ MXene fabricated through wet etching $\mathrm{Ti}_{3} \mathrm{AlC}_{2}$ was employed for designing a screen-printed electrode, which included a dialysis microfluidic chip for multicomponent direct/continuous monitoring of whole blood. ${ }^{58}$ Additionally, composite hydrogels have been designed comprising cellulose and $\mathrm{Ti}_{3} \mathrm{C}_{2}$ MXene, which are endowed with rapid response near-infrared-stimulated features. ${ }^{59}$ The fabricated hydrogels loaded with doxorubicin hydrochloride, showed a remarkable acceleration in the drug release, and were employed as multifunctional nanoplatforms for tumor therapy by intratumoral injection. In this study, MXenes were fabricated via hydrofluoric-acid-etching (HF-etching) of the aluminum (Al) layers in $\mathrm{Ti}_{3} \mathrm{AlC}_{2}$, and then the liquid-phase exfoliation (LPE) in N-methylpyrrolidone (NMP) was performed; the delamination of the prepared bulk $\mathrm{Ti}_{3} \mathrm{C}_{2}$ into few-layer ultrathin $2 \mathrm{D} \mathrm{Ti}_{3} \mathrm{C}_{2}$ MXene nanosheets was accomplished using NMP. ${ }^{59}$

MXene-based materials can also be fabricated via simple and low-cost approaches (e.g., greener methods and solution mixing) into various shapes and morphologies such as fiber, films, and 3D structures. For instance, Shi et al., ${ }^{60}$ have prepared a MXene-chitosan composite via a simple solution technique and utilized the composite for removing $\mathrm{Cr}(\mathrm{vI})$ from aqueous solutions. Additionally, Yan et al. ${ }^{61}$ reported a greener and eco-friendly method for fabricating reduced $\mathrm{Ti}_{3} \mathrm{C}_{2} \mathrm{~T}_{x}$ MXene nanosheets with improved conductivity, oxidation stability, and surface-enhanced Raman spectroscopy (SERS) activity. They have demonstrated a facile and environmentally benign fabrication of MXene-based materials through $\mathrm{L}$-ascorbic acid treatment at room temperature (Fig. 1). ${ }^{61}$

\section{MXenes for tissue engineering and regenerative medicine}

\section{1. $\mathrm{Ti}_{3} \mathrm{C}_{2}$ MXenes}

MXenes have shown promising potential in material sciences and stem cell-based tissue therapies, and thus future multitasked biomedical therapies based on the unique advantages of MXene can be envisioned. ${ }^{62}$ In one study, smart biomaterials comprising titanium carbide $\left(\mathrm{Ti}_{3} \mathrm{C}_{2}\right)$ MXene nanofibers were fabricated for tissue engineering and cell culture. The ensuing hydrophilic composite nanofibers could be prepared via electrospinning and doping due to the abundance of functional hydrophilic groups. ${ }^{62}$ Consequently, the functional groups at the surfaces of MXene nanofiber composites could provide suitable 
(a)

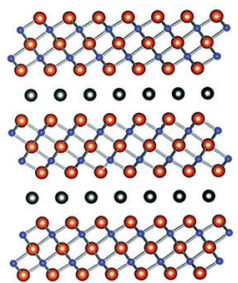

(d) $0^{\circ}$

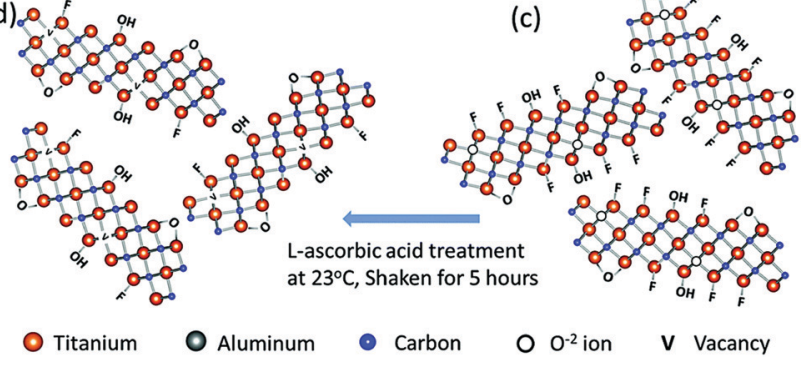

Fig. 1 The fabrication process of reduced $\mathrm{Ti}_{3} \mathrm{C}_{2} \mathrm{~T}_{x}$, including $\mathrm{HF}$ etching ( $a$ and b), delamination (c), and L-ascorbic acid treatment (d) steps. Reproduced from ref. 61 with permission from The Royal Society of Chemistry.

ecosystems for cellular growth. The biochemical representative characteristics have been analyzed by deploying bone marrowderived mesenchymal stem cells (BMSCs); the ensuing MXene composite nanofibers displayed good biocompatibility and remarkably improved cellular activity besides increasing the BMSC's differentiation to osteoblasts. ${ }^{62}$

Smart biomaterials endowed with unique multiple biologic characteristics are ideally suited for tissue engineering and repair. ${ }^{63}$ As an example, zero dimensional (0D) $\mathrm{Ti}_{3} \mathrm{C}_{2}$ MXenebased quantum dots (QDs) were deployed for immunomodulation to improve post-injury tissue repair wherein their inherent immunomodulatory features selectively reduced the human $\mathrm{CD} 4{ }^{+}$IFN- $\gamma^{+}$T-lymphocytes activation while stimulating the expansion of immunosuppressive $\mathrm{CD} 44^{+} \mathrm{CD} 25^{+} \mathrm{FoxP} 3{ }^{+}$regulatory T-cells in a stimulated lymphocyte population; ${ }^{63}$ these $2 \mathrm{D}$ structures with BMSC biocompatibility could stimulate fibroblasts derived from pluripotent stem cells. The incorporation of $\mathrm{Ti}_{3} \mathrm{C}_{2}$ MXene QDs into a hydrogel from chitosan generated a 3D platform with enhanced physicochemical features for the delivery of stem cells and tissue repair; the hydrogel composite showed good conductivity while preserving injectability and thermossensitivity. ${ }^{63}$ On similar lines, the assembly of biocompatible $\mathrm{Ti}_{3} \mathrm{C}_{2} \mathrm{~T}_{z}$-enhanced poly(lactic acid) nanocomposite membranes have been realized where $n$-octyltriethoxysilane mediated the interface of the hydrophobic poly(lactic acid) matrix with the $\mathrm{Ti}_{3} \mathrm{C}_{2} \mathrm{~T}_{z}$ nanosheets; ${ }^{64}$ the final optimized tensile strength of the produced nanocomposite membrane being $72 \mathrm{MPa}, \sim 33 \%$ higher relative to a pure poly(lactic acid) membrane. Thus, the $\mathrm{Ti}_{3} \mathrm{C}_{2} \mathrm{~T}_{z}$ addition could improve the biological attributes of the membrane comprising the osteogenic differentiation of MC3T3E1 mouse preosteoblasts, proliferation, and in vitro adhesion. ${ }^{64}$

The surgical failure and bone-tumor recurrence are of vital concern in view of the residual malignant tumor cells and the

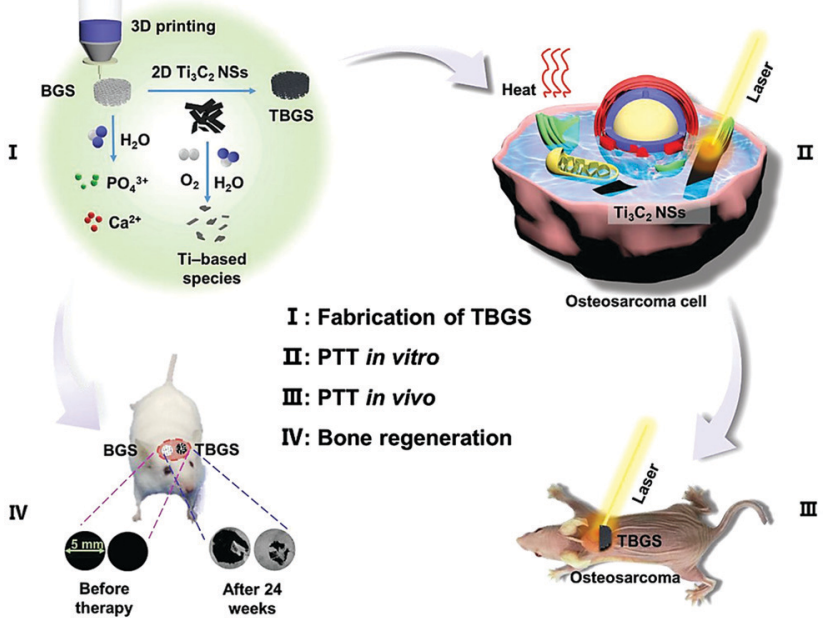

Fig. 2 The process of $\mathrm{Ti}_{3} \mathrm{C}_{2}$-bioactive glass (BG) scaffold (TBGS) preparation (I), bone cancer ablation (PTT in vitro/in vivo) (II and III), and bone tissue regeneration (IV). BGS: bioactive glass scaffold. Reproduced from ref. 65 with permission from Multidisciplinary Digital Publishing Institute (MDPI), (CC BY 4.0).

lack of bone-tissue integration. ${ }^{65}$ Thus, the recurrence and bone deficiency in surgical treatment of malignant bone tumors has generated a demand for producing multifunctional therapeutic platforms for efficient tumor therapy and bone regeneration. As an example, the logical incorporation of $2 \mathrm{D}$ $\mathrm{Ti}_{3} \mathrm{C}_{2}$ MXene with 3D-printing bioactive glass scaffolds has been illustrated wherein simultaneous bone-tumor killing by photonic hyperthermia and bone-tissue regeneration by bioactive scaffolds could be accomplished (Fig. 2). ${ }^{65}$ The ensuing composite scaffolds had ideal attributes for significant photothermal change of integrated $2 \mathrm{D} \mathrm{Ti}_{3} \mathrm{C}_{2}$ MXene for stimulating bone-tumor ablation by near infrared (NIR)-triggered photothermal hyperthermia, achieving whole tumor removal from in vivo bone-tumor xenografts. Remarkably, $\mathrm{Ti}_{3} \mathrm{C}_{2}$ MXeneintegrated composite scaffolds could improve the in vivo growth of newborn bone tissue of the composite bioactive glass scaffolds; such inherent advantages of bone-tumor killing and bone-tissue regeneration may be ideal alternatives for treating bone tumors along with other tissue engineering appliances. ${ }^{65}$

Innovative cell-laden bioink encompassing electroconductive $\mathrm{Ti}_{3} \mathrm{C}_{2}$ MXene nanosheets were created and uniformly dispersed in hydrogels of hyaluronic acid/alginate, comprising an ideally suited operation for extrusion-based 3D bioprinting. ${ }^{66}$ The MXenes containing hydrogel bioinks showed remarkable rheological characteristics, permitting the preparation of layered $3 \mathrm{D}$ structures with shape preservation and significant resolution. Additionally, these nanosheets within the hyaluronic acid/ alginate hydrogels bestowed electrical conductivity to the ink, demonstrating the inadequate electrical conductivity of the present bioinks that misallign with the physicochemical characteristics of the tissue. ${ }^{66}$ Importantly, the condensed human embryonic kidney 293 cells inked by the MXene nanocomposite showed outstanding cell viability (more than 95\%) in both the $3 \mathrm{D}$ bioprinted structures and the bulk hydrogel. ${ }^{66}$ Additionally, 
smart zero-dimensional biocompatible $\mathrm{Ti}_{3} \mathrm{C}_{2} \mathrm{~T}_{x}$ MXene QDs have been prepared with precise surface changes for subcellular nanomedicine applications; ${ }^{67}$ they were spontaneously uptaken into human endothelial cells within 24 hours of cell culture and their localization was remarkably stable with no detectable alterations in cellular morphologies. Furthermore, uptaken MXene QDs showed their significant autofluorescence characteristics at various excitation-emission wavelengths to allow post-delivery evaluations; these bioactive and trackable MXene QDs can be utilized as promising nanosystems for subcellular nanomedicine functions. ${ }^{67}$

Reduced graphene oxide- $\mathrm{Ti}_{3} \mathrm{C}_{2} \mathrm{~T}_{x}$ MXene (rGO-MXene) hydrogel with good hydrophilicity and distinctive inter-linked porous structural design has an unparalleled ability to generate inter-joined three-dimensional networks across three human cell types, namely fibroblast, epithelial adenocarcinoma, and neuroblastoma (Fig. 3). ${ }^{68}$ It has been observed that the cell attachment to rGO-MXene hydrogel was higher than the rGO-control gel alone. In this study, two hydrogels with unique 3D architectural properties were constructed, comprising, rGO-MXene-centered hydrogel (with additional hydrophilic exterior and was adjudged as softer with elastic moduli of $\sim 20 \mathrm{kPa}$ ) and hydrogel based on rGO (endowed with extra hydrophobic shell and was found to be stiffer having elastic moduli of the order of $\sim 40 \mathrm{kPa}){ }^{68}$ The detectable structural self-assembly was quite reminiscent of neuro-spheres from tumorigenic neuron-like neuroblastoma cells after two days on the hydrogel from rGO, which then de-constructed into diffusive lengthened cellular nets subsequent to seven days of culture. Importantly, the constructions of the ensued platforms permitted nutrient diffusion and stimulated the migration of cells all over the hydrogels. ${ }^{68}$ The entire assembly of cultured cells showed remarkable inter-pore dispersion competencies, as established via the extensive generation of migrationimpelled cell cytoskeleton traits (lamellipodia, filopodia, and neurites). Though the in vitro cellular reactions were consistent on such hydrogels, the histocompatibility outlines undoubtedly varied under in vivo conditions or on assorted tissue types; these hydrogels are promising candidates for organ-on-a-chip technologies. ${ }^{68}$
Bone loss or insufficiency is a very challenging issue for the integration of the implant and then functional packing, where evolving biomaterials to supplement bone amount and to regenerate alveolar bone imperfections at the implant site are a critically essential element. To attain innovative inorganic composite materials for the regeneration of bone tissue, MXene can play important and positive roles, especially for the improvement of mechanical features, biocompatibility, and osteoinductivity. ${ }^{64,69}$ In one investigation, nanocomposite membranes based on $2 \mathrm{D} \quad \mathrm{Ti}_{3} \mathrm{C}_{2}$ MXene nanosheets and hydroxyapatite nanowires were constructed with promising potentials for bone regeneration. ${ }^{69}$ The incorporation of ultralong hydroxyapatite nanowires could improve the mechanical characteristics and the hydrophilicity with an improvement in cell adhesion, proliferation, and osteogenic differentiation. Remarkably, this nanocomposite also displayed optimal mechanical features and biological improvements as this MXene-based nanocomposite membrane effectively improved the bone tissue generation in a rat calvarial bone defect (Fig. 4 and 5). ${ }^{69}$ In another study, an electrically conductive MXene hydrogel was constructed with varying compositional features, encompassing delaminated $2 \mathrm{D}$ fluorescent $\mathrm{Ti}_{3} \mathrm{C}_{2}$ nanosheets, bioinspired 3D crosslinked chitosan-based polymeric networks and native $0 \mathrm{D}$ fluorescent carbon dots in honey. ${ }^{70}$ The ensuing hetero-structured $\mathrm{Ti}_{3} \mathrm{C}_{2}$-MXene-honey-chitosan entity showed remarkable permeable construction with controlled degradation and preferred swelling traits; the designed composite was significantly biocompatible with various stem cells, with improved cell attachment and survival characteristics. This introduced electroconductive composite was proposed for various tissue engineering applications. ${ }^{70}$

Remarkably, it was reported that $2 \mathrm{D}$ MXene with $\mathrm{Ti}_{3} \mathrm{C}_{2} \mathrm{~T}_{x}$ composition could nearly quantitatively adsorb urea (initial

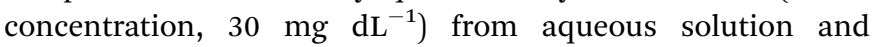
$\sim 94 \%$ from dialysate, the maximal adsorption capacity being $10.4 \mathrm{mg} \mathrm{g}^{-1}$ at ambient temperature. ${ }^{71}$ The analysis at $37{ }^{\circ} \mathrm{C}$ indicated a 2 -fold improvement in urea elimination proficiency from dialysate with the highest urea adsorption amount of $21.7 \mathrm{mg} \mathrm{g}^{-1}$. The designed MXene demonstrated suitable hemocompatibility, with no detectable induction of the cell

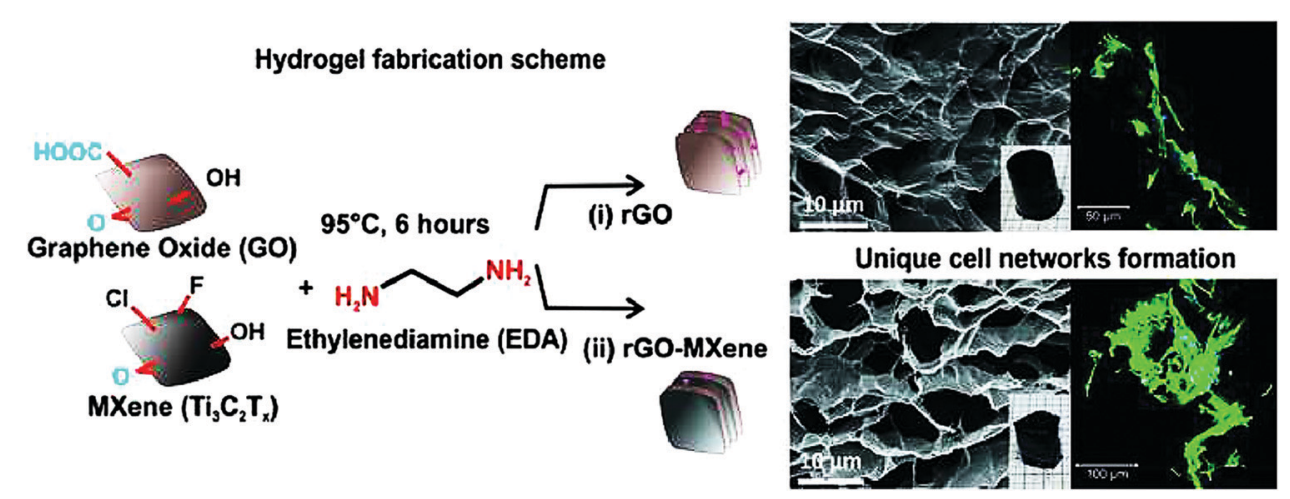

Fig. 3 The formation of a unique cellular network guided by heterostructures based on rGO-MXene hydrogels. Immunofluorescent images of cultured cells were obtained (green fluorescent). Reproduced from ref. 68 with permission from Elsevier, copyright 2020 (CC BY 4.0). 
(a)

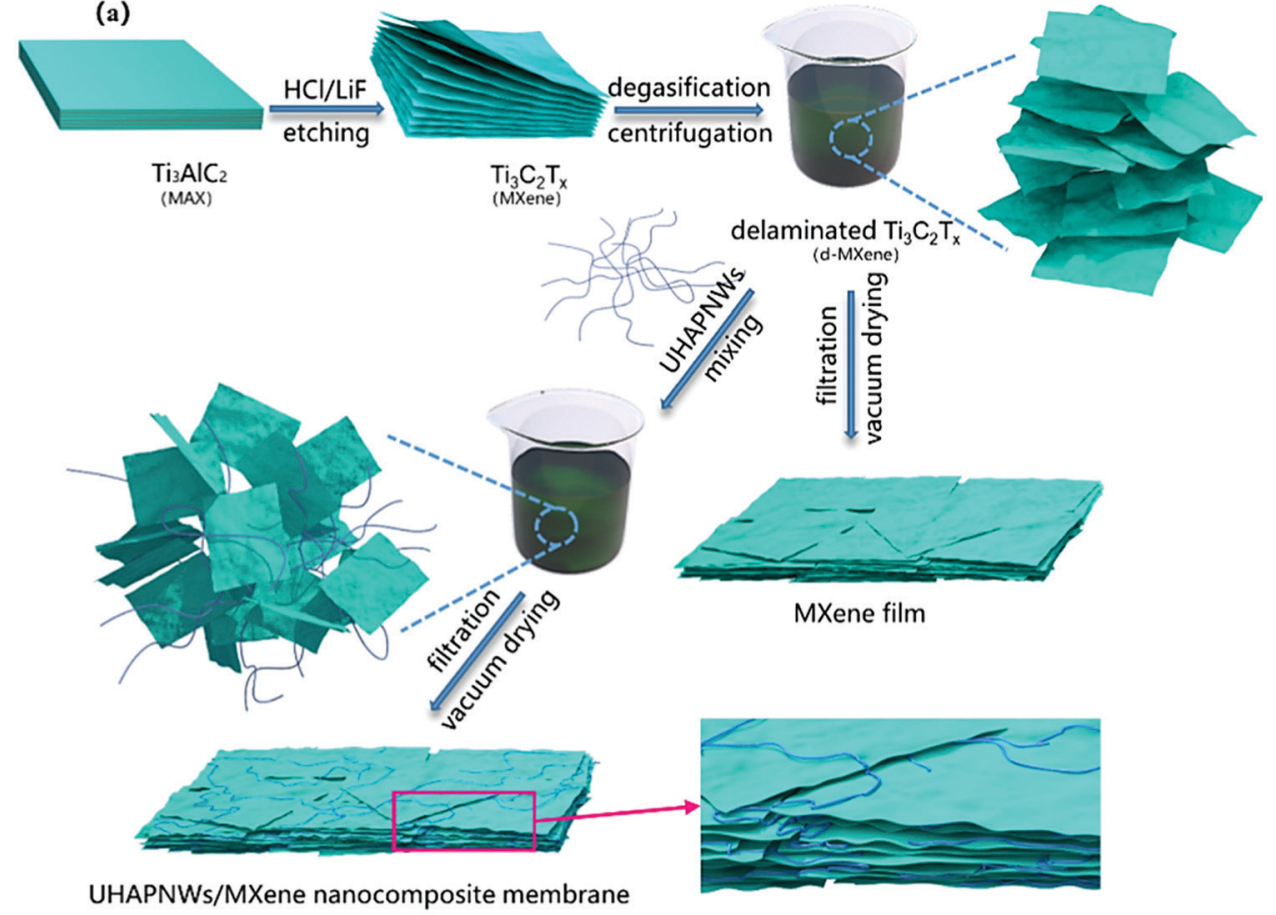

(b)

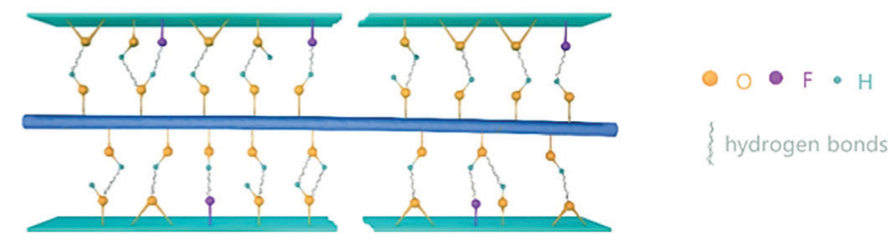

Fig. 4 The preparation of nanocomposite membranes based on 2D MXene nanosheets and hydroxyapatite nanowires (a). The interaction between ultra-long hydroxyapatite nanowires (UHAPNWs) and MXene, involving the hydrogen bonds generated by terminated surface groups of MXene $(-\mathrm{F}$, $=\mathrm{O}$ or $-\mathrm{OH}$ ) and hydroxyl in UHAPNWs (b). Reproduced from ref. 69 with permission from Elsevier, copyright 2020.

apoptosis and reduction of the metabolizing cell fraction, thus revealing no effects on the viability of the cells at concentrations of more than $200 \mu \mathrm{g} \mathrm{mL}^{-1}$. Such MXenes with high biocompatibility and improved selectivity for urea adsorption from dialysate can be exploited in constructing miniaturized dialysate regeneration systems for a wearable artificial kidney. ${ }^{71}$

The electrospun fibers emanating from the polycaprolactone$\mathrm{Ti}_{3} \mathrm{C}_{2}$ MXene composite have been fabricated, and evaluated for their biocompatibility on fibroblast and preosteoblast cell lines; ${ }^{72}$ they were cell-friendly for both cell lines; however, preosteoblast cells showed better cell viability. Additionally, polycaprolactone-MXene composite electrospun fibers with improved wettability, biomineralization, and protein adsorption have been recommended for wound dressing, bone tissue engineering, and also in cancer therapy (Fig. 6). ${ }^{72}$ However, additional studies are warranted for the systematic analysis and functionalization of these materials for broadening their applications, especially in the field of tissue engineering and regenerative medicine.

Synergistic therapeutic strategies for improving the wound repair procedures by coupling electrical stimulation with the hydrogel dressing have been investigated. ${ }^{73}$ Multifunctional hydrogels based on regenerated bacterial cellulose and $\mathrm{Ti}_{3} \mathrm{C}_{2} \mathrm{~T}_{x}$ MXene were designed and commissioned for electrical modulation of the cell behaviors for active skin wound healing under external electrical stimulation. ${ }^{73}$ Consequently, the prepared hydrogels with 2 wt\% MXene exhibited the highest electrical conductivity and the safest biocompatibility. Furthermore, these hydrogels exhibited suitable mechanical features, promising flexibility, high biodegradability, and considerable water-uptake capacity. After in vivo evaluation by using a rat full-thickness defect model, it has been noticed that these hydrogels display better therapeutic effects than the commercial Tegaderm film; in vitro and in vivo results showed that under electrical stimulation, the designed composites could remarkably improve the proliferation activity of NIH3T3 cells, and also enhance the wound healing procedure, as compared to non-electrical stimulation controls. ${ }^{73}$

\section{2. $\mathrm{Nb}_{2} \mathrm{C}$ MXenes}

A multipurpose biomaterial arrangement was constructed by incorporation of $2 \mathrm{D}$ niobium carbide $\left(\mathrm{Nb}_{2} \mathrm{C}\right)$ MXene enveloped 

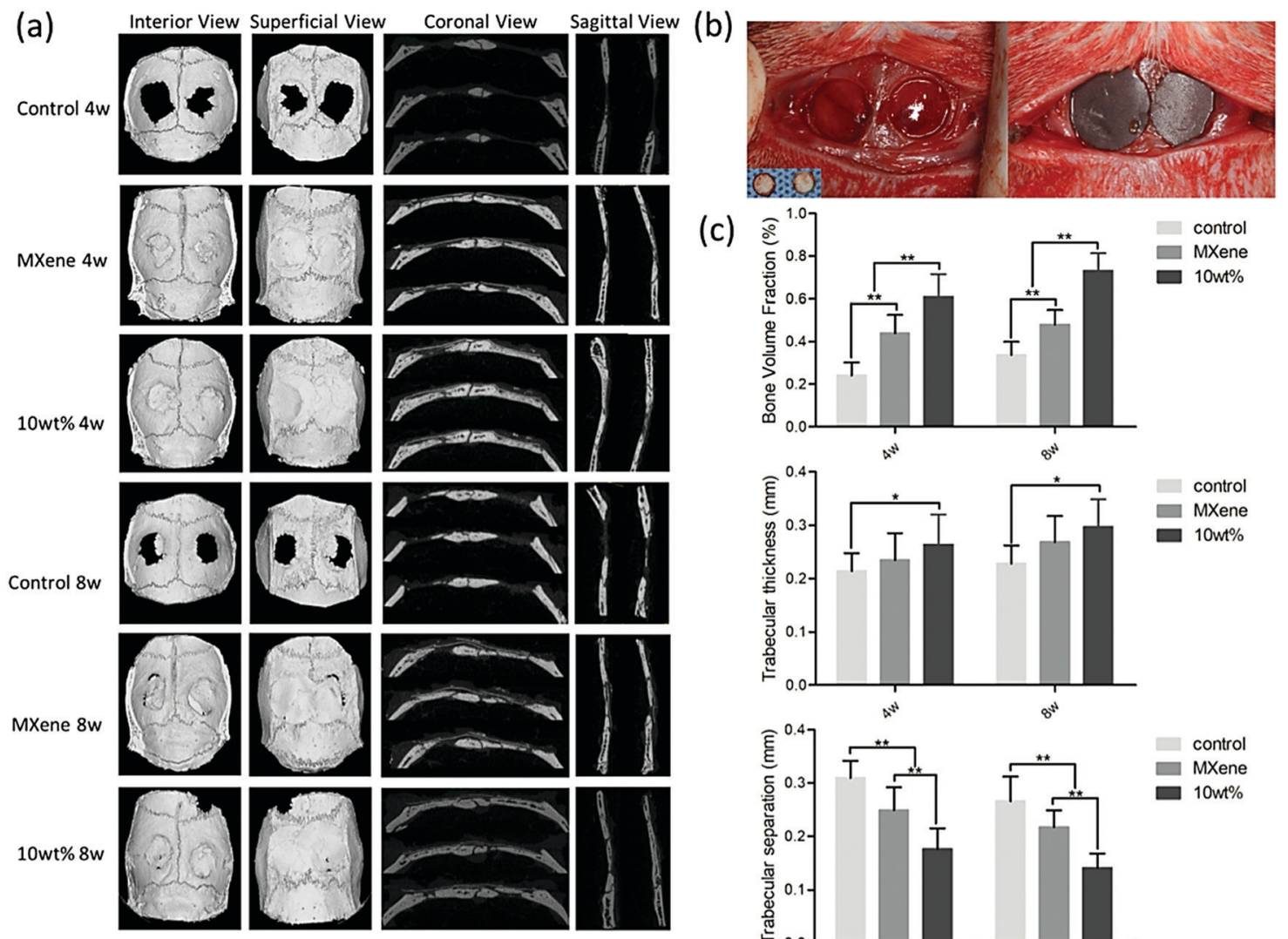

(c)
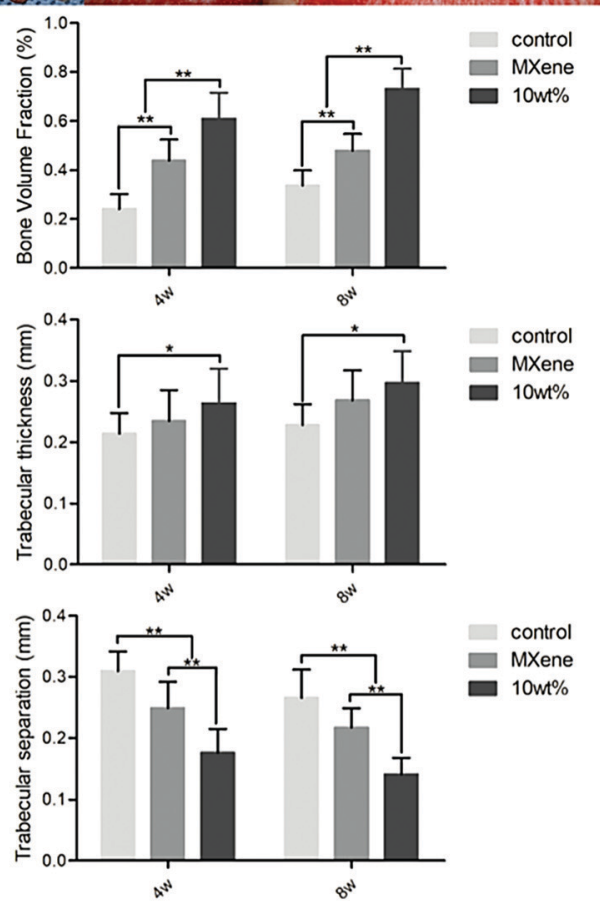

Fig. 5 Bone regeneration performance in the control group, MXene group and ultra-long hydroxyapatite nanowires group (four and eight weeks after the surgery procedure); (a) micro-CT images, (b) surgical process, and (c) radiographic evaluations of bone morphometric factors (bone volume, trabecular thickness, and trabecular separation). Reproduced from ref. 69 with permission from Elsevier, copyright 2020.

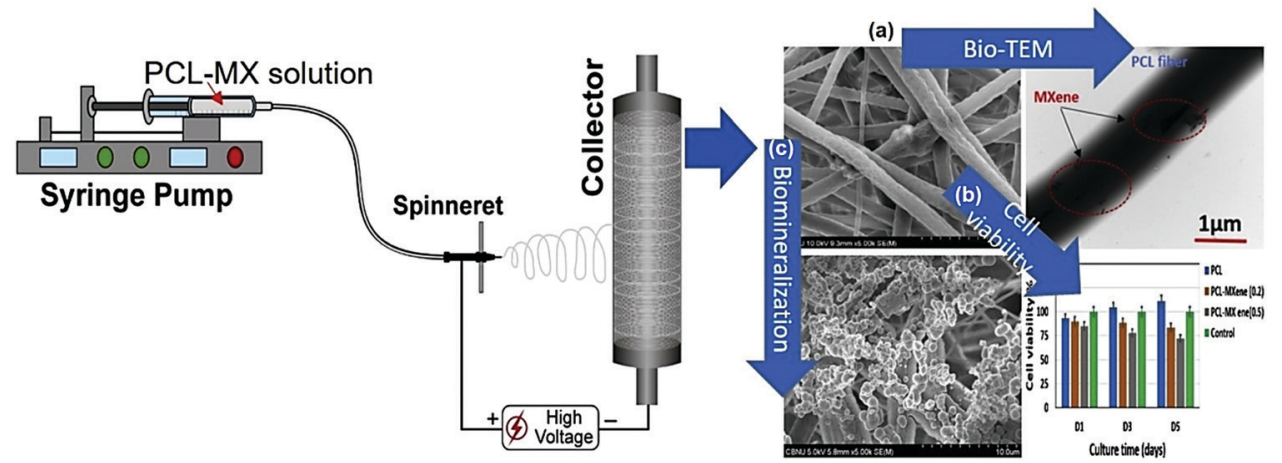

Fig. 6 The process of polycaprolactone-MXene electrospun fiber fabrication and their biocompatibility and cell adhesion study; (a) bio-transmission electron microscopy (TEM), (b) cell viability analysis, and (c) biomineralization process. PCL= polycaprolactone. Reproduced from ref. 72 with permission from Elsevier, copyright 2019.

by $S$-nitrosothiol-grafted mesoporous silica with 3D-printing bioactive glass scaffolds. ${ }^{74}$ Accordingly, the NIR-triggered photonic hyperthermia of MXene in the bio-window (NIR-II) and precisely regulated nitric oxide release could be synchronized for multi-target removal of bone tumors to improve confined osteosarcoma treatment. The generated phosphorus and calcium components in situ from the bioactive glass scaffold stimulated the activity for bone-regeneration, improved by adequate blood supply stimulated by the nitric oxide release on-demand; the adjustable nitric oxide production is critical in sequential adjuvant tumor ablation, combinatorial promotion of coupled vascularization, and bone regeneration. ${ }^{74}$

Interestingly, 2D $\mathrm{Nb}_{2} \mathrm{C}$-based MXene showed suitable potential for in vivo photothermal ablation of mouse tumor 


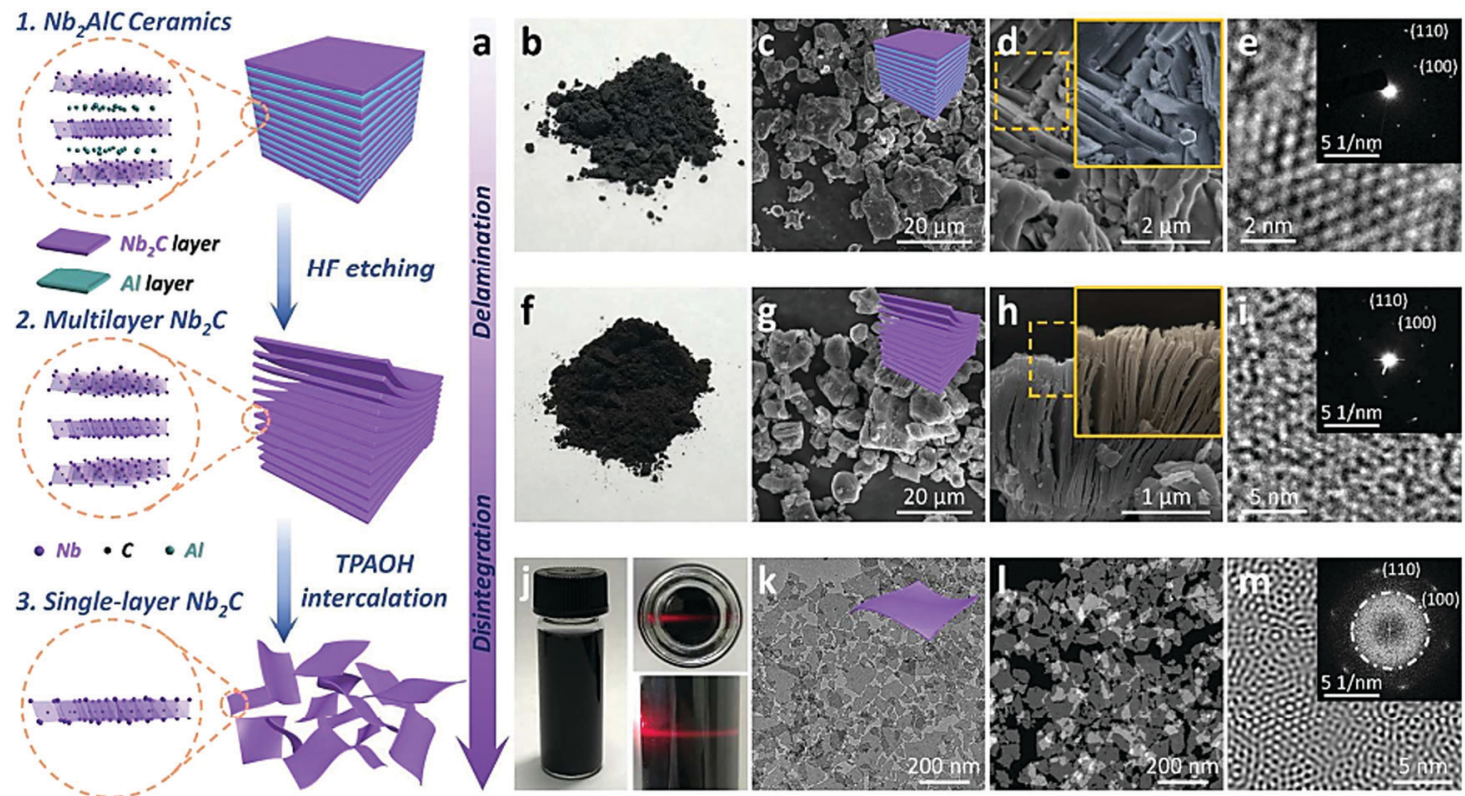

Fig. 7 Ultrathin $\mathrm{Nb}_{2} \mathrm{C}$ nanosheet fabrication (a), digital photograph (b), SEM images (c and d), HRTEM image of $\mathrm{Nb}_{2} \mathrm{AlC}$ ceramic bulks (e), digital photograph (f), SEM images ( $g$ and h), HRTEM image of multilayer $\mathrm{Nb}_{2} \mathrm{C}$ (i). Digital photographs (j), bright-field TEM image (k), dark-field TEM image (l), Fourier transform patterns of single-layer $\mathrm{Nb}_{2} \mathrm{C}(\mathrm{m})$. Reproduced from ref. 75 with permission from American Chemical Society (ACS), copyright 2017.

xenografts in both the NIR-I and NIR-II windows; the ultrathin, lateral-nanosized $\mathrm{Nb}_{2} \mathrm{C}$ nanosheets exhibited remarkable photothermal conversion efficiency $(36.4 \%$ at NIR-I and $45.65 \%$ at NIR-II), and also noteworthy photothermal stability. ${ }^{75}$ These nanosheets had promising features, comprising enzyme-responsive biodegradability to human myeloperoxidase, low phototoxicity, and significant biocompatibility (Fig. 7). ${ }^{75}$

In comparison to graphene, MXenes display hydrophilic behavior with considerable conductivity, providing improved interaction with polymeric matrixes, which bodes well for their utilization in composites. ${ }^{46,76} \mathrm{Zhu}$ et al. ${ }^{77}$ created a soluble 2D MXene $\left(\mathrm{Nb}_{2} \mathrm{C}\right.$ nanosheets) system with polyvinylpyrrolidone microneedles for medical implantation and photothermal destruction of superficial tumors in the NIR-II biological window. Herein, $2 \mathrm{D} \mathrm{Nb}_{2} \mathrm{C}$ nanosheets have been utilized as high-functioning photothermal nano-agents while the biocompatible polyvinylpyrrolidone served as a matrix to preserve the needle structures; the ensued microneedle system had enough skin-permeation capability and unique solubility behavior. After being inserted into the skin of the tumor site, they dissolve readily within a short period to release the loaded nanosheets. These microneedle systems showed high biocompatibility with no remarkable toxicity or side effects, and thus can be exploited for minimally invasive, safe and effective localized therapy. ${ }^{77}$

\section{3. $\quad \mathrm{Ti}_{2} \mathbf{N}$ MXene QDs}

A facile top-down solution phase technique for fabricating nitride-based MXene has been exemplified by titanium nitride $\left(\mathrm{Ti}_{2} \mathrm{~N}\right)$ MXene QDs; ${ }^{78}$ the ensuing $\mathrm{Ti}_{2} \mathrm{~N}$ QDs $(\sim 5 \mathrm{~nm})$ showed remarkable photothermal translation competence under laser irradiation in both the first and second NIR windows, the values being, $808 \mathrm{~nm}, 48.62 \%$ and $1064 \mathrm{~nm}, 45.51 \%$, respectively. ${ }^{78}$ These QDs displayed significant biocompatibility, photoacoustic effect, and photothermal therapy efficiency, in vitro and in vivo. Additionally, they have shown detectable tumor accumulation after four hours of injection, which facilitated photoacoustic imaging-directed photothermal therapy in both bio-windows, NIR-I/II, with no noticeable noxiousness. These QDs endowed with favorable degradation characteristics showed an applicable excretion profile from the body in vivo. Thus, their adequate stability in circulation to achieve therapeutic functions and their smooth elimination from the body bodes well for their applications. ${ }^{78}$

\section{Toxicity and biosafety issues}

MXenes and MXene-based materials with unique properties, including simplistic functionalization procedures, multifunctional potentials, tunable morphologies/structures, biocompatibility, biodegradability, and remarkable physiological stability, can be exploited for various clinical and biomedical applications; ${ }^{76}$ these properties are typical hurdles for most organic entities. ${ }^{33}$ However, toxicity, biosafety, and biocompatibility issues should be further studied by researchers for these 2D MXenes, and also important parameters such as solubility, dispersibility, and long-term toxicity still need to be systematically analyzed. ${ }^{33}$ In one study, the potential toxicity of MXene nanosheets has been evaluated on the early stages of the embryo as well as angiogenesis, and it was indicated that they may produce adverse effects on the primary period of embryogenesis; about $46 \%$ of MXene-exposed embryos died during 1-5 days after exposure. ${ }^{79}$ Additionally, MXenes inhibited the angiogenesis of the embryo's chorioallantoic membrane after five days of incubation at the examined 
concentration. Subsequent analysis of seven regulatory genes of vital significance via reverse transcription polymerase chain reaction (RT-PCR) revealed that these genes were decontrolled in heart, brain, and liver tissues from MXene-treated embryos when compared with their matched controls; thus, more elaborative analytical studies need to be undertaken on the possible toxicity and biosafety of MXenes and MXene-based constructs. ${ }^{79}$

\section{Challenges and future perspectives}

Present investigations in the field of MXenes and MXene-based materials emphasize the importance of controlling their surface properties via surface charge engineering for possible innovative biotechnological or nanomedicine applications. ${ }^{80}$ MXene-based nanoplatforms should have enough biocompatibility and stability in physiological conditions, high targeting capability, selectivity, and controlled release behaviors. ${ }^{59}$ Additionally, there are still some important challenging issues in the development of MXenes and MXene-based materials, especially for their synthesis, dispersion, improvement, better control of surface chemistry functionalization, and producing MXenes with controllable physicochemical properties; accurate characterization techniques for the analysis of functional groups still need to be considered. Importantly, for industrial/ up-scalable production and commercial applications of these materials, the stability and cost-effectiveness issues need to be attended to; there is a vital need for their synthesis via low cost and eco-friendly approaches. ${ }^{44-46}$

The evaluation of biological responses of host tissue cells to the MXene bio-interfaces is very significant and especially should be considered for surface coating of the implants. ${ }^{81}$ As an example, the cytocompatibility of selected MAX phases, $\mathrm{Ti}_{3} \mathrm{AlC}_{2}, \mathrm{Ti}_{3} \mathrm{SiC}_{2}$, and $\mathrm{Ti}_{2} \mathrm{AlN}$, have been systematically analyzed, in vitro. ${ }^{82}$ These phases were found to be anoxic to fibroblasts and preosteoblasts; relative to the strong viable fibroblasts, varied cellular responses for them could be detected for preosteoblasts. It was revealed that under an osteoblastic environment, $\mathrm{Ti}_{2} \mathrm{AlN}$ had better cell proliferation and differentiation performance than the other surveyed materials. Remarkably, relative to the partly oxidized $\mathrm{TiC}_{x} \mathrm{O}_{y}$ layer on $\mathrm{Ti}_{3} \mathrm{AlC}_{2}$ and $\mathrm{Ti}_{3} \mathrm{SiC}_{2}$, the partially oxidized $\operatorname{TiN}_{x} \mathrm{O}_{y}$ layer on the $\mathrm{Ti}_{2} \mathrm{AlN}$ showed a remarkable affinity for the $\mathrm{Ca}^{2+}$ ions, and thus useful cytocompatibility of $\mathrm{Ti}_{2} \mathrm{AlN}$ was detected. ${ }^{82}$ Another study described that the nanostructured $\mathrm{Ti}_{3} \mathrm{C}_{2}$ layer could protect the bulk titanium implant against the harsh conditions of biological tissues as well as stimulate the proliferation, adhesion, and osteoblast activity. Furthermore, it could provide better boneimplant contacts for the implant compared to the uncoated titanium implant. ${ }^{83}$

Undeniably, MXenes with their unique physicochemical properties have demonstrated several biomedical potentials, especially for bioimaging, biosensing, cancer therapy/diagnosis, antibacterial activity, drug delivery, tissue engineering, and regenerative medicine. ${ }^{84,85}$ But, for clinical applications of MXenes and MXene-based materials, there are still various

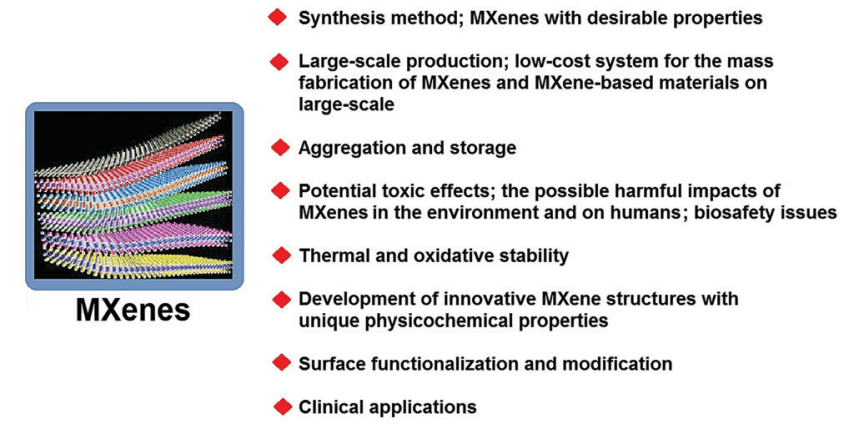

Fig. 8 Important challenges associated with the fabrication and applications of MXenes.

challenging issues, especially regarding their biosafety (shortterm and long-term toxicity) and their therapeutic efficacy (especially for their potential in synergistic combinational therapy together with conventional strategies) (Fig. 8). ${ }^{86,87}$ Indeed, these 2D structures for clinical applications should have enough homogeneity and preferably demonstrate suitable and efficient functions with acceptable similarity in patients. Additionally, their clinical potentials are related to their sufficient cytocompatibility/ biocompatibility, stability (long-lasting suitable effects), and good biodegradability. These critical properties are affected by some important factors such as size, compositions, surface features, and surface modification/functionalization. An acceptable pharmacokinetic balance should exist between the degradation and stability of MXenes, improving their clinical therapeutic efficacy and reducing possible adverse side effects. ${ }^{81,88}$

MXenes have great potential for drug delivery/release and can be deployed as efficient platforms for synergistic chemophotothermal ablation of cancers. However, their controlled and targeted release behaviors should be analytically evaluated and optimized. In one study, an innovative heterostructured nanocarrier based on $\mathrm{Ti}_{3} \mathrm{C}_{2}$ MXenes and cobalt nanowires was constructed for synergistic chemo-photothermal cancer therapy with dual stimuli-responsive drug release and magnetic controlling capabilities. ${ }^{89}$ Additionally, Li et al. ${ }^{90}$ have constructed a composite nanoplatform using MXenes for synergistic chemophotothermal therapy of hepatocellular carcinoma. These materials with efficient surface chemistry for targeting engineering have exhibited suitable drug release/delivery capacity, improved hydrophilicity, and acceptable dispersity. ${ }^{90}$

\section{Conclusion and future outlooks}

In recent years, MXenes have shown remarkable prospects in biomedical applications, especially in tissue engineering, owing to their ultrathin structural features and specific morphologies with a range of extraordinary physicochemical, electronic, optical and biological properties. The creation of multi purposeful scaffolds with distinctive attributes of wellregulated discharge behavior, greatly proficient photothermal translation and stimulatory tissue regeneration offers a fascinating biomaterial stage for the varied treatment of assorted diseases and malignancies such as cancers and tumors. 
These 2D materials can be fabricated by applying facile and economic procedures and can be easily functionalized for multiplex biomedical appliances. Their surface properties can be tailored for specific applications such as tissue engineering, energy storage, imaging, sensors, and water filtration, by adjusting synthetic aspects and deploying optimized conditions; MXene possesses high biocompatibility and can be constructed for anticancer, antibacterial, and immunomodulatory purposes. In this context, polymer functionalized MXene nanocomposites have shown significant photothermal conversion efficiency, selectivity, and stimuli-responsiveness towards malignant cells, electron sensitivity, and remarkable antimicrobial characteristics.

Future investigations are warranted on responsive scaffolds (both, mechanical and electrical) for regenerative medicine, intracellular fluorescent probes, partition membranes for artificial organs, and implantation of bio-electronic peripheries for neurotransmitter and bio-electronic signal recognition; remarkable electrical and optical features of MXenes render them ideal candidates for sensing applications. Additionally, innovative sustainable and greener synthetic methods for ecofriendly fabrication of MXenes as well as their functional modification for innovative tissue engineering applications need to be explored. There is a vital need to establish the influences of the size and composition of MXenes on their properties and to analyze the interactions between MXenes and drugs or cells; toxicity, biodegradability biocompatibility, histopathological and biosafety issues need to be systematically and analytically evaluated. Suitable biofunctionalization and chemical surface modifications can help to fine-tune various aspects, namely the functionality, stability, bioavailability, biodegradability and biocompatibility, reduce the adverse effects/ immune system reactions and increase their targeting property.

\section{Conflicts of interest}

The author(s) declare no competing interest.

\section{References}

1 F. J. O’Brien, Mater. Today, 2011, 14, 3.

2 J. R. Gershlak, S. Hernandez, G. Fontana, L. R. Perreault, K. J. Hansen, S. A. Larson, B. Y. K. Binder, D. M. Dolivo, T. Yang, T. Dominko, M. W. Rolle, P. J. Weathers, F. MedinaBolivar, C. L. Cramer, W. L. Murphy and G. R. Gaudette, Biomaterials, 2017, 125, 13-22.

3 G. Jamalipour Soufi and S. Iravani, Green Chem., 2020, 22, 2662-2687.

4 S. Iravani and R. S. Varma, Green Chem., 2019, 21, 4839-4867.

5 S. Iravani and R. S. Varma, Green Chem., 2020, 22, 612-636.

6 S. Iravani and R. S. Varma, Environ. Chem. Lett., 2020, 18, 703-727.

7 M. Naguib, V. N. Mochalin, M. W. Barsoum and Y. Gogotsi, Adv. Mater., 2014, 26, 992-1005.
8 M. Naguib, M. Kurtoglu, V. Presser, J. Lu, J. Niu, M. Heon, L. Hultman, Y. Gogotsi and M. W. Barsoum, Adv. Mater., 2011, 23, 4248-4253.

9 Z. Xie, S. Chen, Y. Duo, Y. Zhu, T. Fan, Q. Zou, M. Qu, Z. Lin, J. Zhao, Y. Li, L. Liu, S. Bao, H. Chen, D. Fan and H. Zhang, ACS Appl. Mater. Interfaces, 2019, 11, 22129-22140.

10 X.-Y. Feng, B.-Y. Ding, W.-Y. Liang, F. Zhang, T.-Y. Ning, J. Liu and H. Zhang, Laser Phys. Lett., 2018, 15, 085805.

11 C. Wang, Y. Wang, X. Jiang, J. Xu, W. Huang, F. Zhang, J. Liu, F. Yang, Y. Song, Y. Ge, Q. Wu, M. Zhang, H. Chen, J. Liu and H. Zhang, Adv. Opt. Mater., 2019, 7, 1900060.

12 Q. Wu, S. Chen, Y. Wang, L. Wu, X. Jiang, F. Zhang, X. Jin, Q. Jiang, Z. Zheng, J. Li, M. Zhang and H. Zhang, Adv. Mater. Technol., 2019, 4, 1800532.

13 Y. Zhang, X. Jiang, J. Zhang, H. Zhang and Y. Li, Biosens. Bioelectron., 2019, 130, 315-321.

14 X. Zhan, C. Si, J. Zhou and Z. Sun, Nanoscale Horiz., 2020, 5, 235-258.

15 G. K. Nasrallah, M. Al-Asmakh, K. Rasool and K. A. Mahmoud, Environ. Sci.: Nano, 2018, 5, 1002-1011.

16 H. Lin, S. Gao, C. Dai, Y. Chen and J. Shi, J. Am. Chem. Soc., 2017, 139, 16235-16247.

17 K. Rasool, M. Helal, A. Ali, C. E. Ren, Y. Gogotsi and K. A. Mahmoud, ACS Nano, 2016, 10, 3674-3684.

18 K. Huang, Z. Li, J. Lin, G. Han and P. Huang, Chem. Soc. Rev., 2018, 47, 5109-5124.

19 C. Dai, H. Lin, G. Xu, Z. Liu, R. Wu and Y. Chen, Chem. Mater., 2017, 29, 8637-8652.

20 A. Jastrzębska, E. Karwowska, D. Basiak, A. Zawada, W. Ziemkowska, T. Wojciechowski, D. Jakubowska and A. Olszyna, Int. J. Electrochem. Sci., 2017, 12, 2159-2172.

21 A. Szuplewska, D. Kulpińska, A. Dybko, M. Chudy, A. Maria Jastrzębska, A. Olszyna and Z. Brzózka, Trends Biotechnol., 2020, 38, 264-279.

22 Y. Wang, W. Feng and Y. Chen, Chin. Chem. Lett., 2020, 31, 937-946.

23 Y. Bai, K. Zhou, N. Srikanth, J. H. Pang, X. He and R. Wang, RSC Adv., 2016, 6, 35731-35739.

24 V. N. Borysiuk, V. N. Mochalin and Y. Gogotsi, Nanotechnology, 2015, 26, 265705.

25 M. Khazaei, M. Arai, T. Sasaki, A. Ranjbar, Y. Liang and S. Yunoki, Phys. Rev. B: Condens. Matter Mater. Phys., 2015, 92, 075411.

26 Y. Liu, H. Xiao and W. A. Goddard, J. Am. Chem. Soc., 2016, 138, 15853-15856.

27 G. Gao, G. Ding, J. Li, K. Yao, M. Wu and M. Qian, Nanoscale, 2016, 8, 8986-8994.

28 C. Si, J. Zhou and Z. Sun, ACS Appl. Mater. Interfaces, 2015, 7, 17510-17515.

29 G. Wang, J. Phys. Chem. C, 2016, 120, 18850-18857.

30 J. He, P. Lyu and P. Nachtigall, J. Mater. Chem. C, 2016, 4, 11143-11149.

31 M. Han, K. Maleski, C. E. Shuck, Y. Yang, J. T. Glazar, A. C. Foucher, K. Hantanasirisakul, A. Sarycheva, N. C. Frey, S. J. May, V. B. Shenoy, E. A. Stach and Y. Gogotsi, J. Am. Chem. Soc., 2020, 142, 19110-19118. 
32 X. Jiang, A. V. Kuklin, A. Baev, Y. Ge, H. Ågren, H. Zhang and P. N. Prasad, Phys. Rep., 2020, 848, 1-58.

33 H. Lin, Y. Chen and J. Shi, Adv. Sci., 2018, 5, 1800518.

34 A. Zavabeti, A. Jannat, L. Zhong, A. A. Haidry, Z. Yao and J. Z. Ou, Nano-Micro Lett., 2020, 12, 66.

35 L. Wang, P. Hu, Y. Long, Z. Liu and X. He, J. Mater. Chem. A, 2017, 5, 22855-22876.

36 S. Chen, C. Xing, D. Huang, C. Zhou, B. Ding, Z. Guo, Z. Peng, D. Wang, X. Zhu, S. Liu, Z. Cai, J. Wu, J. Zhao, Z. Wu, Y. Zhang, C. Wei, Q. Yan, H. Wang, D. Fan, L. Liu, H. Zhang and Y. Cao, Sci. Adv., 2020, 6, eaay6825.

37 W. Cheng, X. Zeng, H. Chen, Z. Li, W. Zeng, L. Mei and Y. Zhao, ACS Nano, 2019, 13, 8537-8565.

38 X. Zeng, M. Luo, G. Liu, X. Wang, W. Tao, Y. Lin, X. Ji, L. Nie and L. Mei, Adv. Sci., 2018, 5, 1800510.

39 M. Luo, T. Fan, Y. Zhou, H. Zhang and L. Mei, Adv. Funct. Mater., 2019, 29, 1808306.

40 Z. Xie, M. Peng, R. Lu, X. Meng, W. Liang, Z. Li, M. Qiu, B. Zhang, G. Nie, N. Xie, H. Zhang and P. N. Prasad, Light: Sci. App., 2020, 9, 161.

41 W. Tao, X. Zhu, X. Yu, X. Zeng, Q. Xiao, X. Zhang, X. Ji, X. Wang, J. Shi, H. Zhang and L. Mei, Adv. Mater., 2017, 29, 1603276.

42 J. Zhu, G. S. Xiao and X. X. Zuo, Nano-Micro Lett., 2020, 12, 120.

43 J. Zhang, Y. Fu and A. Mo, Int. J. Nanomed., 2019, 14, 10091-10103.

44 J. Yang, M. Naguib, M. Ghidiu, L. M. Pan, J. Gu, J. Nanda, J. Halim, Y. Gogotsi and M. W. Barsoum, J. Am. Ceram. Soc., 2016, 99, 660-666.

45 P. Eklund, J. Rosen and P. O. Å. Persson, J. Phys. D: Appl. Phys., 2017, 50, 113001.

46 R. M. Ronchi, J. T. Arantes and S. F. Santos, Ceram. Int., 2019, 45, 18167-18188.

47 J. Yang, W. Bao, P. Jaumaux, S. Zhang, C. Wang and G. Wang, Adv. Mater. Interfaces, 2019, 6, 1802004.

48 E. A. Mayerberger, R. M. Street, R. M. McDaniel, M. W. Barsoum and C. L. Schauer, RSC Adv., 2018, 8, 35386-35394.

49 Z. Liu, M. Zhao, H. Lin, C. Dai, C. Ren, S. Zhang, W. Peng and Y. Chen, J. Mater. Chem. B, 2018, 6, 3541-3548.

50 P. Karthikeyan, S. S. Elanchezhiyan, J. Preethi, K. Talukdar, S. Meenakshi and C. M. Park, Ceram. Int., 2021, 47, 732-739.

51 Y. Gogotsi and B. Anasori, ACS Nano, 2019, 13, 8491-8494.

52 L. Ma, L. R. L. Ting, V. Molinari, C. Giordano and B. S. Yeo, J. Mater. Chem. A, 2015, 3, 8361-8368.

53 C. Xu, L. Wang, Z. Liu, L. Chen, J. Guo, N. Kang, X.-L. Ma, H.-M. Cheng and W. Ren, Nat. Mater., 2015, 14, 1135-1141.

54 P. Urbankowski, B. Anasori, T. Makaryan, D. Er, S. Kota, P. L. Walsh, M. Zhao, V. B. Shenoy, M. W. Barsoum and Y. Gogotsi, Nanoscale, 2016, 8, 11385.

55 T. Li, L. Yao, Q. Liu, J. Gu, R. Luo, J. Li, X. Yan, W. Wang, P. Liu and B. Chen, Angew. Chem., Int. Ed., 2018, 57, 6115-6119.

56 W. Sun, S. Shah, Y. Chen, Z. Tan, H. Gao, T. Habib, M. Radovic and M. Green, J. Mater. Chem. A, 2017, 5, 21663-21668.

57 O. Salim, K. A. Mahmoud, K. K. Pant and R. K. Joshi, Mater. Today Chem., 2019, 14, 100191.
58 J. Liu, X. Jiang, R. Zhang, Y. Zhang, L. Wu, W. Lu, J. Li, Y. Li and H. Zhang, Adv. Funct. Mater., 2019, 29, 1807326.

59 C. Xing, S. Chen, X. Liang, Q. Liu, M. Qu, Q. Zou, J. Li, H. Tan, L. Liu, D. Fan and H. Zhang, ACS Appl. Mater. Interfaces, 2018, 10, 27631-27643.

60 H. Wan, L. Nan, H. Geng, W. Zhang and H. Shi, Processes, 2021, 9, 524 .

61 T. B. Limbu, B. Chitara, J. D. Orlando, M. Y. Garcia Cervantes, S. Kumari, Q. Li, Y. Tang and F. Yan, J. Mater. Chem. C, 2020, 8, 4722-4731.

62 R. Huang, X. Chen, Y. Dong, X. Zhang, Y. Wei, Z. Yang, W. Li, Y. Guo, J. Liu, Z. Yang, H. Wang and L. Jin, ACS Appl. Bio Mater., 2020, 3, 2125-2131.

63 A. Rafieerad, W. Yan, G. L. Sequiera, N. Sareen, E. Abu-ElRub, M. Moudgil and S. Dhingra, Adv. Healthcare Mater., 2019, 8, 1900569.

64 K. Chen, Y. Chen, Q. Deng, S.-H. Jeong, T.-S. Jang, S. Du, H.-E. Kim, Q. Huang and C.-M. Han, Mater. Lett., 2018, 229, 114-117.

65 S. Pan, J. Yin, L. Yu, C. Zhang, Y. Zhu, Y. Gao and Y. Chen, Adv. Sci., 2020, 7, 1901511.

66 H. Rastin, B. Zhang, A. Mazinani, K. Hassan, J. Bi, T. T. Tung and D. Losic, Nanoscale, 2020, 12, 16069-16080.

67 A. Rafieerad, W. Yan, A. Amiri and S. Dhingra, Mater. Des., 2020, 196, 109091.

68 J. K. Wychowaniec, J. Litowczenko, K. Tadyszak, V. Natu, C. Aparicio, B. Peplińska, M. W. Barsoum, M. Otyepka and B. Scheibe, Acta Biomater., 2020, 115, 104-115.

69 Y. Fu, J. Zhang, H. Lin and A. Mo, Mater. Sci. Eng., C, 2021, 118, 111367.

70 A. Rafieerad, G. L. Sequiera, W. Yan, P. Kaur, A. Amiri and S. Dhingra, J. Mech. Behav. Biomed. Mater., 2020, 101, 103440.

71 F. Meng, M. Seredych, C. Chen, V. Gura, S. Mikhalovsky, S. Sandeman, G. Ingavle, T. Ozulumba, L. Miao, B. Anasori and Y. Gogotsi, ACS Nano, 2018, 12, 10518-10528.

72 G. P. Awasthi, B. Maharjan, S. Shrestha, D. P. Bhattarai, D. Yoon, C. H. Park and C. S. Kim, Colloids Surf., A, 2020, 586, 124282.

73 L. Mao, S. Hu, Y. Gao, L. Wang, W. Zhao, L. Fu, H. Cheng, L. Xia, S. Xie, W. Ye, Z. Shi and G. Yang, Adv. Healthcare Mater., 2020, 2000872, DOI: 10.1002/adhm.202000872.

74 Q. Yang, H. Yin, T. Xu, D. Zhu, J. Yin, Y. Chen, X. Yu, J. Gao, C. Zhang, Y. Chen and Y. Gao, Small, 2020, 16, 1906814.

75 H. Lin, S. Gao, C. Dai, Y. Chen and J. Shi, J. Am. Chem. Soc., 2017, 139, 16235-16247.

76 S. M. George and B. Kandasubramanian, Ceram. Int., 2020, 46, 8522-8535.

77 S. Lin, H. Lin, M. Yang, M. Ge, Y. Chen and Y. Zhu, Nanoscale, 2020, 12, 10265-10276.

78 J. Shao, J. Zhang, C. Jiang, J. Lin and P. Huang, Chem. Eng. J., 2020, 400, 126009.

79 H. Alhussain, R. Augustine, E. A. Hussein, I. Gupta, A. Hasan, A. E. Al Moustafa and A. Elzatahry, J. Biomed. Nanotechnol., 2020, 16, 364-372.

80 A. Rozmysłowska-Wojciechowska, J. Mitrzak, A. Szuplewska, M. Chudy, J. Woźniak, M. Petrus, T. Wojciechowski, A. S. Vasilchenko and A. M. Jastrzębska, Materials, 2020, 13, 2347. 
81 M. Soleymaniha, M.-A. Shahbazi, A. R. Rafieerad, A. Maleki and A. Amir, Adv. Healthcare Mater., 2019, 8, 1801137.

82 K. Chen, N. Qiu, Q. Deng, M.-H. Kang, H. Yang, J.-U. Baek, Y.-H. Koh, S. Du, Q. Huang and H.-E. Kim, ACS Biomater. Sci. Eng., 2017, 3, 2293-2301.

83 F. Veronesi, G. Giavaresi, M. Fini, G. Longo, C. A. Ioannidu, A. S. Abusco, F. Superti, G. Panzini, C. Misiano, A. Palattella, P. Selleri, N. Girolamo, D. V. Garbarino and L. Politi, Mater. Sci. Eng., C, 2017, 70, 264-271.

84 L. Gao, C. Li, W. Huang, S. Mei, H. Lin, Q. Ou, Y. Zhang, J. Guo, F. Zhang, S. Xu and H. Zhang, Chem. Mater., 2020, 32, 1703-1747.
85 W. Huang, L. Hu, Y. Tang, Z. Xie and H. Zhang, Adv. Funct. Mater., 2020, 30, 2005223.

86 M. Qiu, A. Singh, D. Wang, J. Qu, M. Swihart, H. Zhang and P. N. Prasad, Nanotoday, 2019, 25, 135-155.

87 H. Huang, R. Jiang, Y. Feng, H. Ouyang, N. Zhou, X. Zhang and Y. Wei, Nanoscale, 2020, 12, 1325-1338.

88 Z. Xie, T. Fan, J. An, W. Choi, Y. Duo, Y. Ge, B. Zhang, G. Nie, N. Xie, T. Zheng, Y. Chen, H. Zhang and J. S. Kim, Chem. Soc. Rev., 2020, 49, 8065-8087.

89 Y. Liu, Q. Han, W. Yang, X. Gan, Y. Yang, K. Xie, L. Xie and Y. Deng, Mater. Sci. Eng., C, 2020, 116, 111212.

90 Z. Li, H. Zhang, J. Han, Y. Chen, H. Lin and T. Yang, Adv. Mater., 2018, 30, 1706981. 